\title{
Order-Independent Algorithm for the Asymptotic Stability of Complex Polynomials
}

\author{
Ziad Zahreddine \\ Correspondence: Mathematics Division, College of Engineering, Kuwait College of Science and Technology, Doha \\ Area, 7th Ring Road, Kuwait
}

Received: May 10, 2021 Accepted: August 25, 2021 Online Published: September 22, 2021

doi:10.5539/jmr.v13n5p24

URL: https://doi.org/10.5539/jmr.v13n5p24

\begin{abstract}
The Extended Routh Array (ERA) settles the asymptotic stability of complex polynomials. The ERA is a natural extension of the Routh Array which applies only to real polynomials. Although the ERA is a nice theoretical algorithm for stability testing, it has its limitations. Unfortunately, as the order of the polynomial increases, the size of calculations increases dramatically as will be shown below. In the current work, we offer an alternative algorithm which is basically equivalent to the ERA, but has the extra advantage of being simpler, more efficient, and easy to apply even to large order polynomials. In all the steps required in the construction of the new algorithm, only one single and simple algebraic operation is needed, which makes it a polynomial order-independent algorithm.
\end{abstract}

2010 AMS Subject Classification: Primary 37C75, 93C15. Secondary 93D, 30E

Keywords: Hurwitz polynomials, asymptotic stability, routh array, extended routh array

\section{Introduction}

The problem of determining conditions under which all the roots of a given polynomial lie in the left-half plane is one of the fundamental problems in the study of stability of a dynamic system. Such polynomials are called Hurwitz polynomials, or asymptotically stable polynomials, and they arise in a variety of applications such as control systems, circuit analysis, numerical computations, systems theory, and digital signal processing to name a few. For some references in this respect see (Gutman, 1979), (Krein \& Neimark, 1981) and (Zahreddine, 1992).

Most of the existing methods for testing the Hurwitz stability are restricted to the real case. For a variety of references in this context, see (Howland, 1971), (Lipatov \& Sokolov, 1979), and (Zahreddine, 1999). Since modern communication and information theory uses both complex signals and complex envelopes in signal analysis and detection, it is becoming more and more imperative to address the theory of stability essentially when dealing with systems having complex coefficients. With this in mind, the Extended Routh Array (ERA) which is the complex counterpart of the Routh Array for real systems was developed in (Zahreddine, 1993). The ERA settles the Hurwitz stability of complex polynomials.

In (Zahreddine, 1994), we introduced the concept of wide sense stability and we showed how the ERA can handle the appearance of vanishing leading array elements (singularities).

For more references in this context, see (Barnett, 1995), (Zahreddine, 2003), (Zahreddine, 2018), and (Zahreddine, 2019).

The problem of root distribution of a polynomial in some sub-regions of the complex plane like sectors, ellipses, parabolas has also been investigated (Gutman, 1979), and (Zahreddine, 1996).

Most of the approaches used in the derivation of stability criteria involve elaborate techniques such as: index theory, Sturm chains, Rouche's theorem, Lyaponov equations, or generalized bezoutians. See for example (Heinig \& Jungnickel, 1984) (Horn \& Johnson, 1991) and (Mawhin, 1997). In the same vein, the ERA also suffers a major defect. As the order of the polynomial increases, the size of calculations increases dramatically as will be made clear in Section 2 below.

In the current work, we address this deficiency by introducing a more efficient algorithm which is essentially equivalent to the ERA. In constructing the new algorithm, basic algebraic operations are used which consist only of calculations of $2 \times 2$ determinants. Therefore, the new algorithm is simpler, more efficient, and polynomial order-independent, which makes it easily applicable even to large order polynomials.

In section 2, we reconsider the construction of the ERA and we recall some major facts about asymptotic stability of complex polynomials. In section 3, we offer an alternative form for the ERA which will lead in Section 4 to the new order-independent algorithm. We show the equivalence of the new algorithm with the ERA, as well as its advantages over 
it. We end up in Section 5 by some concluding remarks.

\section{The Extended Routh Array (ERA)}

The definitions and results stated in this section are based on (Zahreddine, 1993) and (Zahreddine, 2017).

Definition 2.1

A non-constant polynomial is a Hurwitz polynomial if all its roots have negative real parts.

\section{Definition 2.2}

The paraconjugate of a rational function $f(s)$ is defined by $f^{*}(s)=\overline{f(-\bar{s})}$, where $\bar{s}$ denotes the complex conjugate of $s$.

For example, if

$$
f(s)=s^{n}+a_{1} s^{n-1}+\cdots+a_{n-2} s^{2}+a_{n-1} s+a_{n},
$$

then

$$
f^{*}(s)=(-1)^{n} s^{n}+(-1)^{n-1} \bar{a}_{1} s^{n-1}+\cdots+\bar{a}_{n-2} s^{2}-\bar{a}_{n-1} s+\bar{a}_{n} .
$$

Also, if $f$ is written in the factored form

$$
f(s)=\left(s-s_{1}\right)\left(s-s_{2}\right) \cdots\left(s-s_{n}\right)
$$

then its paraconjugate can be written as

$$
f^{*}(s)=(-1)^{n}\left(s+\overline{s_{1}}\right)\left(s+\overline{s_{2}}\right) \cdots\left(s+\overline{s_{n}}\right)
$$

\section{Definition 2.3}

Define the function

Obviously, $h$ can be written as

$$
h(s)= \begin{cases}\frac{f-f^{*}}{f+f^{*}} & \text { if } n \text { odd } \\ \frac{f+f^{*}}{f-f^{*}} & \text { if } n \text { even }\end{cases}
$$

$$
h(s)=\frac{s^{n}+i \operatorname{Im} a_{1} s^{n-1}+\operatorname{Re} a_{2} s^{n-2}+i \operatorname{Im} a_{3} s^{n-3}+\operatorname{Re} a_{4} s^{n-4}+\cdots}{\operatorname{Re} a_{1} s^{n-1}+i \operatorname{Im} a_{2} s^{n-2}+\operatorname{Re} a_{3} s^{n-3}+i \operatorname{Im} a_{4} s^{n-4} \cdots}
$$

This function is sometimes referred to as the test fraction (Hovstad, 1989).

Call $h_{1}$ and $h_{2}$ the numerator and denominator of $h$ respectively. If $\operatorname{Re} a_{1} \neq 0$, denote by $h_{3}$ the remainder of the division of $h_{1}$ by $h_{2}$, and in general denote by $h_{k}$ the remainder obtained upon dividing $h_{k-2}$ by $h_{k-1}$ for $k=3, \ldots, n+1$. It is worthwhile to mention that Lemma 4.1 of [Zahreddine, 1993) gives direct expressions of the coefficients of $h_{k}$ in terms of those of $h_{k-1}$ by $h_{k-2}$. Now, the ERA is defined by: 


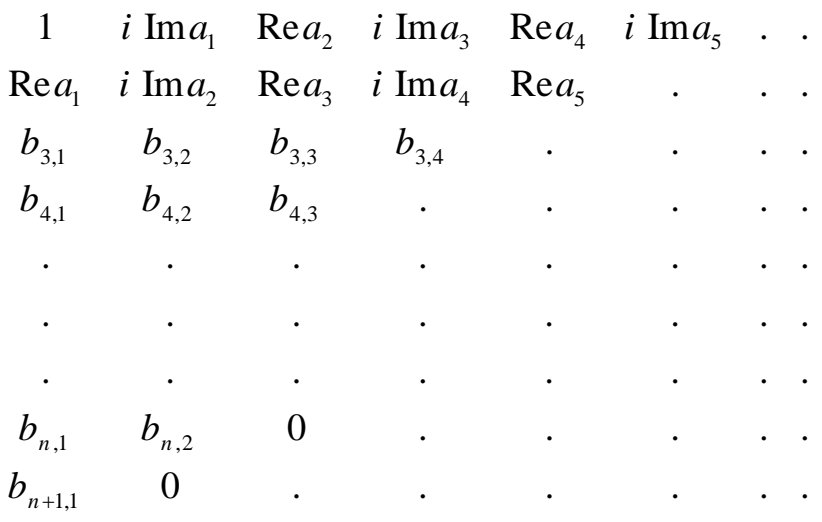

where

$$
\begin{aligned}
& b_{3,1}=\frac{1}{\operatorname{Re} a_{1}}\left(\operatorname{Re} a_{1} \operatorname{Re} a_{2}-\operatorname{Re} a_{3}\right)-\frac{i \operatorname{Im} a_{2}}{\left(\operatorname{Re} a_{1}\right)^{2}}\left(i \operatorname{Re} a_{1} \operatorname{Im} a_{1}-i \operatorname{Im} a_{2}\right), \\
& b_{3,2}=\frac{1}{\operatorname{Re} a_{1}}\left(i \operatorname{Re} a_{1} \operatorname{Im} a_{3}-i \operatorname{Im} a_{4}\right)-\frac{\operatorname{Re} a_{3}}{\left(\operatorname{Re} a_{1}\right)^{2}}\left(i \operatorname{Re} a_{1} \operatorname{Im} a_{1}-i \operatorname{Im} a_{2}\right), \\
& b_{4,1}=\frac{1}{b_{3,1}}\left(b_{3,1} \operatorname{Re} a_{3}-\operatorname{Re} a_{1} b_{3,3}\right)-\frac{b_{3,2}}{b_{3,1}^{2}}\left(i b_{3,1} \operatorname{Im} a_{2}-\operatorname{Re} a_{1} b_{3,2}\right), \\
& b_{4,2}=\frac{1}{b_{3,1}}\left(i b_{3,1} \operatorname{Im} a_{4}-\operatorname{Re} a_{1} b_{3,4}\right)-\frac{b_{3,3}}{b_{3,1}^{2}}\left(i b_{3,1} \operatorname{Im} a_{2}-\operatorname{Re} a_{1} b_{3,2}\right),
\end{aligned}
$$

and so on.

In the above ERA, the $k$-th row represents the coefficients of $h_{k}$ for $k=1,2,3, \ldots, n+1$ and all rows have the same size by completing by zeros.

Theorem 4.1 of (Zahreddine, 1993) shows that the ERA is an algorithm to test the asymptotic stability of complex polynomials.

Theorem 2.1 (Theorem 4.1, (Zahreddine, 1993))

The complex polynomial $f$ in (1) is Hurwitz if and only if each term of the first column of the extended Routh array is positive.

\section{An Alternative Form of the ERA}

Let's consider the following arrangement, in which the $1^{\text {st }}$ and $2^{\text {nd }}$ rows match those of the ERA. The notations used in this arrangement will be justified in due context.

$$
\begin{aligned}
& \begin{array}{llllllll}
p_{0,1} & p_{0,2} & p_{0,3} & p_{0,4} & p_{0,5} & p_{0,6}
\end{array} . \\
& \begin{array}{lllll}
p_{1,1} & p_{1,2} & p_{1,3} & p_{1,4} & p_{1,5}
\end{array} \\
& \begin{array}{llll}
q_{1,1} & q_{1,2} & q_{1,3} & q_{1,4}
\end{array} \\
& \begin{array}{llll}
p_{2,1} & p_{2,2} & p_{2,3} & p_{2,4}
\end{array} \\
& q_{2,1} \quad q_{2,2} \quad q_{2,3} \\
& p_{3,1} \quad p_{3,2} \quad p_{3,3}
\end{aligned}
$$

where

$$
p_{0,1}=1, \quad p_{0, k}=\left\{\begin{array}{ll}
\operatorname{Re} a_{k-1} & k>2 \& \text { odd } \\
i \operatorname{Im} a_{k-1} & k \text { even }
\end{array}, \quad p_{1, k}=\left\{\begin{array}{cc}
\operatorname{Re} a_{k} & k \text { odd } \\
i \operatorname{Im} a_{k} & k \text { even }
\end{array}\right.\right.
$$

and 


$$
\begin{aligned}
& q_{1,1}=\frac{p_{1,1} p_{0,2}-p_{0,1} p_{1,2}}{p_{1,1}}, \quad q_{1,2}=\frac{p_{1,1} p_{0,3}-p_{0,1} p_{1,3}}{p_{1,1}}, \quad q_{1,3}=\frac{p_{1,1} p_{0,4}-p_{0,1} p_{1,4}}{p_{1,1}}, \ldots \\
& p_{2,1}=\frac{p_{1,1} q_{1,2}-q_{1,1} p_{1,2}}{p_{1,1}}, \quad p_{2,2}=\frac{p_{1,1} q_{1,3}-q_{1,1} p_{1,3}}{p_{1,1}}, \quad p_{2,3}=\frac{p_{1,1} q_{1,4}-q_{1,1} p_{1,4}}{p_{1,1}}, \ldots \\
& q_{2,1}=\frac{p_{2,1} p_{1,2}-p_{1,1} p_{2,2}}{p_{2,1}}, \quad q_{2,2}=\frac{p_{2,1} p_{1,3}-p_{1,1} p_{2,3}}{p_{2,1}}, \quad q_{2,3}=\frac{p_{2,1} p_{1,4}-p_{1,1} p_{2,4}}{p_{2,1}}, \ldots \\
& p_{3,1}=\frac{p_{2,1} q_{2,2}-q_{2,1} p_{2,2}}{p_{2,1}}, \quad p_{3,2}=\frac{p_{2,1} q_{2,3}-q_{2,1} p_{2,3}}{p_{2,1}}, \quad p_{3,3}=\frac{p_{2,1} q_{2,4}-q_{2,1} p_{2,4}}{p_{2,1}}, \ldots
\end{aligned}
$$

The next theorem shows that the above array can well serve as an algorithm to test the Hurwitness of a complex polynomial.

Theorem 3.1

The complex polynomial $f$ in (1) is Hurwitz if and only if $p_{k 1}>0$ for all $k, 1 \leq k \leq n$.

Proof

Suppose the polynomial $f(s)=s^{n}+a_{1} s^{n-1}+\cdots+a_{n-2} s^{2}+a_{n-1} s+a_{n}$ given in (1) is Hurwitz, then by Theorem 3.2 (Zahreddine, 1993), the function $h(s)$ given in (2) above can be written as

$$
\begin{aligned}
& h(s)=a_{0}+b_{0} s+\frac{1}{a_{1}+b_{1} s+\frac{1}{a_{2}+b_{2} s+}} \\
& +\frac{1}{a_{n-2}+b_{n-2} s+\frac{1}{a_{n-1}+b_{n-1} s}}
\end{aligned}
$$

where $\operatorname{Re} a_{j}=0$, and $b_{j}>0$ for $0 \leq j \leq n-1$.

Lemma 4.1 (Zahreddine, 1993) shows how the $b_{k}$ 's in the above expression can be written in terms of the elements of the $1^{\text {st }}$ column of the ERA. In fact,

$$
b_{j}=\frac{b_{j+1,1}}{b_{j+2,1}}
$$

for $0 \leq j \leq n-1$, and where we assume that $b_{1,1}=1$ and $b_{2,1}=\operatorname{Re} a_{1}$.

Also by Lemma 4.1 (Zahreddine, 1993), the polynomials $f_{1}, f_{2}, \ldots, f_{n+1}$ participating in the construction of the ERA satisfy the following relations:

$$
f_{j+1}=\left(a_{j}+b_{j} s\right) f_{j+2}+f_{j+3} \text {, for } 0 \leq j \leq n-1 \text {, and where } f_{n+2}=0 .
$$

Those relations are a particular case of Lemma 4.1 of (Zahreddine, 1993), and it can easily be seen that the terms arising from these relations are the same as those forming our new algorithm (3). That leads to the following expression of $h(s)$, 


$$
\begin{gathered}
h(s)=\alpha_{0}+\beta_{0} s+\frac{1}{\alpha_{1}+\beta_{1} s+\frac{1}{\alpha_{2}+\beta_{2} s+}} \\
\vdots \\
+\frac{1}{\alpha_{n-2}+\beta_{n-2} s+\frac{1}{\alpha_{n-1}+\beta_{n-1} s}}
\end{gathered}
$$

Where

$$
\beta_{j}=\frac{p_{j, 1}}{p_{j+1,1}} \text { for } 0 \leq j \leq n-1 .
$$

The uniqueness of the continued fraction expansion of $h(s)$ implied by Section 3 of (Zahreddine, 1993) leads to the conclusion that $b_{j}=\beta_{j}$ for $0 \leq j \leq n-1$.

We want to prove the relations $p_{j, 1}=b_{j+1,1}$ for $0 \leq j \leq n-1$, where we define $b_{1,1}=1$ and $b_{2,1}=\operatorname{Re} a_{1}$.

Obviously these relations hold for $j=0$, and $j=1$, since $p_{0,1}=1$ and $p_{1,1}=\operatorname{Re} a_{1}$ by (4).

Now the relation $b_{1}=\beta_{1}$ combined with (5) and (6) leads to $\frac{b_{2,1}}{b_{3,1}}=\frac{p_{1,1}}{p_{2,1}}$.

And since $b_{2,1}=p_{1,1}=\operatorname{Re} a_{1}$, it follows that $p_{2,1}=b_{3,1}$.

Therefore the relation $p_{j, 1}=b_{j+1,1}$ is also proved for $j=2$.

We proceed by induction and we consider the induction assumption to be

$$
p_{j-1,1}=b_{j, 1} \text { for some } j, 3 \leq j \leq n \text {. }
$$

Then $\beta_{j-1}=\frac{p_{j-1,1}}{p_{j, 1}}$ by (6) and $b_{j-1}=\frac{b_{j, 1}}{b_{j+1,1}}$ by (5).

The relations $\beta_{j-1}=b_{j-1} \quad$ (uniqueness of the continued fraction expansion) and $p_{j-1,1}=b_{j, 1}$ (Induction assumption), lead to the conclusion that $p_{j, 1}=b_{j+1,1}$

and that proves our claim that $p_{j, 1}=b_{j+1,1}$ for $0 \leq j \leq n-1$.

By Theorem 4.1 (Zahreddine, 1993), $b_{j+1,1}>0$ for $1 \leq j \leq n$, it therefore follows that $p_{j, 1}>0$ for $1 \leq j \leq n$, and that completes the proof of the theorem.

\section{Order-Independent Algorithm}

We introduce the following arrangement based on the ERA, and arrangement (3) defined in Section 3. 


\begin{tabular}{ccc|cc|cc}
$\operatorname{Re} a_{1}$ & $i \operatorname{Im} a_{2}$ & $\operatorname{Re} a_{3}$ & $i \operatorname{Im} a_{4}$ & $\operatorname{Re} a_{5}$ & $i \operatorname{Im} a_{6}$ &. \\
\cline { 1 - 5 } 1 & $i \operatorname{Im} a_{1}$ & $\operatorname{Re} a_{2}$ & $i \operatorname{Im} a_{3}$ & $\operatorname{Re} a_{4}$ & $i \operatorname{Im} a_{5}$ &. \\
0 & $\operatorname{Re} a_{1}$ & $i \operatorname{Im} a_{2}$ & $\operatorname{Re} a_{3}$ & $i \operatorname{Im} a_{4}$ & $\operatorname{Re} a_{5}$ &. \\
\cline { 1 - 5 } 0 & 1 & $i \operatorname{Im} a_{1}$ & $\operatorname{Re} a_{2}$ & $i \operatorname{Im} a_{3}$ & $\operatorname{Re} a_{4}$ &.. \\
0 & 0 & $\operatorname{Re} a_{1}$ & $i \operatorname{Im} a_{2}$ & $\operatorname{Re} a_{3}$ & $i \operatorname{Im} a_{4}$ &. \\
\cline { 1 - 5 } & 0 & 1 & $i \operatorname{Im} a_{1}$ & $\operatorname{Re} a_{2}$ & $i \operatorname{Im} a_{3}$ &..
\end{tabular}

\section{Theorem 4.1}

The complex polynomial $f$ in (1) is Hurwitz if and only if

$$
(-1)^{j(j-1) / 2} D_{j}>0
$$

for $1 \leq j \leq n$ and where $D_{1}, D_{2}, \ldots, D_{n}$ are the first $n$ minors highlighted in arrangement (7) above.

Proof

Suppose the polynomial $f(s)=s^{n}+a_{1} s^{n-1}+\cdots+a_{n-2} s^{2}+a_{n-1} s+a_{n}$ given in (1) is Hurwitz,

then by Theorem 3.1 of Section 3

$$
p_{j 1}>0
$$

for $0 \leq j \leq n$.

From the $2 k^{\text {th }}$ row, subtract $1 / \operatorname{Re} a_{1}$ multiplied by the $(2 k-1)^{\text {st }}$ row for $1 \leq k \leq j-1$.

Using Arrangement 3 of Section (3), we get

$$
D_{j}=p_{11}\left|\begin{array}{ccccc}
q_{11} & q_{12} & q_{13} & \cdot & \cdot \\
p_{11} & p_{12} & p_{13} & \cdot & \cdot \\
0 & q_{11} & q_{12} & \cdot & \cdot \\
0 & p_{11} & p_{12} & \cdot & \cdot \\
0 & 0 & q_{11} & \cdot & \cdot \\
\cdot & \cdot & \cdot & \cdot & \cdot
\end{array}\right|
$$

where evidently

$$
p_{11}=\operatorname{Re} a_{1}, p_{12}=i \operatorname{Im} a_{2}, p_{13}=\operatorname{Re} a_{3} \text { and so on. }
$$

Obviously, the newly defined determinant $D_{j}$ is of order $2 j-1$ for $2 \leq \mathrm{j} \leq n$.

Again, from the $(2 k-1)^{\text {st }}$ row, subtract $q_{11} / p_{11}$ multiplied by the $2 k^{\text {th }}$ row for $1 \leq k \leq j-1$.

Also, using Arrangement 3 of Section (3), we get

$$
D_{j}=(-1)^{j-1} p_{11}^{2} D_{j-1}^{(1)} \text {, for } 2 \leq j \leq n .
$$

where $D_{r}^{(k)}$ represents the determinan t $D_{r}$ where both subscripts of each of its elements are augmented by $k$. We then conclude that

$$
D_{j}=(-1)^{j(j-1) / 2} p_{11}^{2} p_{21}^{2} \ldots p_{(j-1) 1}^{2} p_{j 1}
$$


which can also be written as

$$
(-1)^{j(j-1) / 2} D_{j}=p_{11}^{2} p_{21}^{2} \ldots p_{(j-1) 1}^{2} p_{j 1} \text {, for } 2 \leq j \leq n .
$$

By Theorem 3.1 above, we conclude that

$$
p_{j 1}>0 \text {, for } 0 \leq j \leq n \text {. }
$$

Hence

$$
(-1)^{j(j-1) / 2} D_{j}>0, \text { for } 1 \leq j \leq n .
$$

For the converse, assume that

$$
(-1)^{j(j-1) / 2} D_{j}>0, \text { for } 1 \leq j \leq n .
$$

If $j=1$, then $D_{1}=p_{11}>0$.

In the formula $(-1)^{j(j-1) / 2} D_{j}=p_{11}^{2} p_{21}^{2} \ldots p_{(j-1) 1}^{2} p_{j 1}$, for $2 \leq j \leq n$, put $j=2$, then $p_{21}>0$.

If we continue with this process of induction, we get

$$
p_{j 1}>0, \text { for } 1 \leq j \leq n,
$$

and by Theorem 3.1, the complex polynomial $f$ in (1) is Hurwitz and that completes the proof.

\section{Concluding Remarks}

The Extended Routh Array (ERA) settles the asymptotic stability of complex polynomials. They are sometimes called Hurwitz polynomials. However, the ERA remains more of a theoretical algorithm rather that an applicable tool for stability testing. A new equivalent algorithm is devised which is simpler and more efficient. the new algorithm is polynomial order-independent, since no matter how large the order of the polynomial is, the construction of the new algorithm involves at every step the calculation of a $2 \times 2$ determinant.

\section{References}

Barnett, S. (1995). Some modern applications of mathematics. Prentice Hall.

Gutman, S. (1979). Root clustering of a complex matrix in an algebraic region. IEEE Transactions on Automatic Control, 24(4), 647-650. https://doi.org/10.1109/TAC.1979.1102109

Heinig, G., \& Jungnickel, U. (1984). On the Routh-Hurwitz and Schur-Cohn Problems for Matrix Polynomials and Generalized Bezoutians. Mathematische Nachrichten, 116(1), 185-196. https://doi.org/10.1002/mana.19841160114

Horn, R. A., Horn, R. A., \& Johnson, C. R. (1994). Topics in matrix analysis. Cambridge university press.

Hovstad, R. M. (1989). A short proof of a continued fraction test for the stability of polynomials. Proceedings of the American Mathematical Society, 76-79. https://doi.org/10.1090/S0002-9939-1989-0973839-7

Howland, J. L. (1971, May). The method of quadratic forms in dynamic stability problems. In Third Canadian Conference in Applied Mechanics (Calgary).

Krein, M. G., \& Naimark, M. A. (1981). The method of symmetric and Hermitian forms in the theory of the separation of the roots of algebraic equations. Linear and multilinear algebra, 10(4), 265-308. https://doi.org/10.1080/03081088108817420

Lipatov, A. V. (1979). Some sufficient conditions for stability and instability of continuous liner stationary systems translated form Automaticai Telemekhanika, No. 9, 30/37. Automat. Remote Cont., 39, 1285-1291.

Mawhin, J. (1997). Some remarks on the \$lOmega \$-stability for families of polynomials. Archivum Mathematicum, 33(1), 139-145.

Zahreddine, Z. (1992). Explicit relationships between Routh-Hurwitz and Schur-Cohn types of stability. Irish Math. Soc. Bull, 29(49-54), 27-2. 
Zahreddine, Z. (1993). An extension of the Routh array for the asymptotic stability of a system of differential equations with complex coefficients. Applicable Analysis, 49(1-2), 61-72. https://doi.org/10.1080/00036819308840165

Zahreddine, Z. (1994). Wide sense stability of complex systems of differential equations of arbitrary dimension

Zahreddine, Z. (1996). On the $\{\$ \backslash$ Gamma $\$\}$-stability of systems of differential equations in the Routh-Hurwitz and the Schur-Cohn cases. Bulletin of the Belgian Mathematical Society-Simon Stevin, 3(3), 363-368. https://doi.org/10.36045/bbms/1105554392

Zahreddine, Z. (2003). Alternative Approaches to the General Stability Problem. In Conference in Mathematical Analysis \& Applications, American University of Sharjah.

Zahreddine, Z. (2017). Parallel Properties of Poles of Positive Functions and those of Discrete Reactance Functions. International Journal of Mathematical Analysis, 11(24), 1141-1150. 77106

Zahreddine, Z. (2018). On positive para-odd and complex discrete reactance functions. Journal of Interdisciplinary Mathematics, 21(1), 243-251. https://doi.org/10.1080/09720502.2017.1367525

Zahreddine, Z. (2019). New Versions of the Hermite Bieler Theorem in Stability Contexts. American Journal of Applied Mathematics, 7(1), 1-4. https://doi.org/10.11648/j.ajam.20190701.11

Zahreddine, Z. I. A. D. (1999). On some properties of Hurwitz polynomials with application to stability theory. Soochow Journal of Mathematics, 25(1), 19-28.

\section{Copyrights}

Copyright for this article is retained by the author(s), with first publication rights granted to the journal.

This is an open-access article distributed under the terms and conditions of the Creative Commons Attribution license (http://creativecommons.org/licenses/by/4.0/). 